\title{
ARTEMETHER LOADED ETHYLCELLULOSE NANOSUSPENSIONS: EFFECTS OF FORMULATION VARIABLES, PHYSICAL STABILITY AND DRUG RELEASE PROFILE
}

\author{
NASRIN E. KHALIFA, ABUBAKR O. NUR*, ZUHEIR A. OSMAN \\ Department of Pharmaceutics, Faculty of Pharmacy, University of Khartoum \\ Email: abubakr100@hotmail.com
}

Received: 07 Mar 2017 Revised and Accepted: 20 Apr 2017

\section{ABSTRACT}

Objective: The aim of this study was to explore the individual and joint effects of drug: ethylcellulose ratio, content of tween 80 and chloroform: water volume ratio on particles' size and size distribution of artemether loaded ethyl cellulose nanosuspension formulations, aiming to achieve nanosuspension with desired particles properties, stability and drug release profile.

Methods: Mixed levels design was used to generate a series of artemether loaded ethylcellulose nanosuspensions that produced by emulsificationsolvent evaporation technique. Formulations were qualified for particle size and size distribution using dynamic light scattering technique. Best ranked formulation was then evaluated for stability and drug release rate and kinetics.

Results: Drug: polymer ratio, content of surfactant and organic: water volume ratio were found to exert considerable influences (p<0.05) on particle size of produced nanosuspensions, either individually or as joint variables. Peak intensity property of nanosuspensions was found to be influenced by drug: polymer ratio $(\mathrm{p}<0.05)$ whereas the influences of different variables on the polydisperse index property appear inconsequential ( $\mathrm{p}>0.05)$. Best ranked (optimal) artemether nanosuspension proved stable and capable to improve and maintain the release of loaded drug over 24 $\mathrm{h}$, at least under the setting conditions of this study.

Conclusion: Focusing on both the individual and joint influences of formulation variables assist in achieving nanosuspension with desired particles characteristics, stability and drug release profile.

Keywords: Artemether nanosuspensions, Effects of variables, Dynamic light scattering, Particle size, Size distribution, Stability, Drug release

(C) 2017 The Authors. Published by Innovare Academic Sciences Pvt Ltd. This is an open access article under the CC BY license (http://creativecommons.org/licenses/by/4.0/) DOI: http://dx.doi.org/10.22159/ijpps.2017v9i6.18321

\section{INTRODUCTION}

The improved drug bioavailability offered by nanosizing technique can be explained in terms of the increased saturation solubility and/or the increased dissolution velocity of nanosized drugs. Moreover, the technique provides an excellent choice of administering lipophilic drugs via different routes [1]

Nanosuspensions have become one of the most active areas of research in the field of drug delivery in consequence to their ability to deliver drugs to the right place, at appropriate times and in the right dosage. Therefore, numerous reports addressing applications of nanosuspension as a novel drug delivery system for many drugs through different routes of drug administration have been reviewed in the literature [2].

Artemether, a model drug in this study, is a poorly water soluble anti-malarial drug that measures low bioavailability after oral administration [3] and many attempts have been made to improve the aqueous solubility of the drug including solid dispersion and inclusion complexes techniques $[4,5]$.

Many reports have focused on the characterization and qualifications of the formulated nanosuspension products [6, 7] whilst others have documented the individual effects of formulation or processing variables on the attributes of nanosuspensions of different drugs $[8,9]$. However, the joint influences of formulation variables on particles properties of nanosuspensions have received less recognition in the relevant literature. The present study entails application of mixed levels full factorial designs to explore the individual and joint effects of drug: ethylcellulose ratio, content of tween 80 and ratio of chloroform: water volume on particles' size and size distribution of artemether loaded ethyl cellulose nanosuspension formulations, aiming to determine best setting for each variable to achieve a nanosuspension drug formulation with desired particles properties. Moreover, the study was designed in a way to investigate short term stability and drug release characteristics of a selected nanosuspension formulation that measured optimum particle size, size distribution and dispersity characteristics.

\section{MATERIALS AND METHODS}

\section{Materials}

Artemether was a pharmaceutical grade product of Chemiino Co., P. R. China. Ethyl cellulose (pharmaceutical grade) and Tween 80 (400$500 \mathrm{cS}$; $50 \mathrm{Sp}$ value) were products of Sd fine-chem Ltd, India. Chloroform was analytical grade product of Applichem Co. Germany. Other materials and reagents were either analytical or pharmaceutical grade obtained from different commercial sources.

Preparation of artemether loaded ethylcellulose Nano suspensions

For initial screening and subsequent optimization purposes, mixed levels factorial design was selected to generate 18 nanosuspension formulations using varying level settings for three formulation variables, namely, drug: polymer ratio, tween 80 loading level and ratio of chloroform: water volume (table 1).

Nanosuspension formulations were prepared by emulsificationsolvent evaporation technique [10]. For each run, artemether (100 $\mathrm{mg}$ ) and the respective amount of ethylcellulose were accurately weighed and dissolved in $5 \mathrm{ml}$ of chloroform. Obtained solution was poured into the relevant amount of distilled water maintained at 25 ${ }^{\circ} \mathrm{C}$ and containing specified amount of tween 80 . The yielded mixture was stirred by an ultra-sonicator (400 Watt Homogenizer/Omni International, USA) for $1 \mathrm{~min}$ to form microemulsion. The microemulsion was then continuously stirred on magnetic stirrer (Decibel Instruments, India) for $4 \mathrm{~h}$ to allow the volatile solvent to evaporate and the respective artemether loaded ethylcellulose nanosuspensions was thus obtained. 


\section{Characterization of particles in different nanosuspension formulations}

Produced artemether loaded ethylcellulose nanosuspensions in the design were subjected to particles characterization and measurements. The mean particle size, \% peak intensity (PI \%) and polydisperse index (PDI) for the formulations were determined by Dynamic Light Scattering (DLS) with a Zetasizer (Nano ZS-90, Malvern Instruments Ltd., UK) equipped with the data transfer software (DTS). The reading was carried out at a 900 angle with respect to the incident beam. Moreover, best ranked (optimized) drug loaded ethylcellulose nanosuspension formulation was qualified for short term stability and in vitro drug release.

Influences of formulation variables on attributes of drug nanosuspensions

Based on the changing profiles of the measured nanosuspensions properties (particle size, \% PI and PDI) as a result of variation in level settings of investigated variables, model fitting approaches were utilized to investigate for the possible individual and joint effects of drug: polymer ratio, chloroform: water volume ratio and Tween 80 loading level on particles size and size distribution characteristics of different nanosuspension formulations.

\section{Formulations assortment and ranking optimization}

A composite index (CI) comprises of 3 preset constraints for the responses particle size $(10-400 \mathrm{~nm}), \%$ PI $(85-115 \%)$ and PDI (0.05-0.45) was applied to rank the produced artemether loaded ethylcellulose nanosuspensions in consequence to their displayed responses. For each response, demonstrated value was transformed to a value between 0 and 33.33 using suitable linear regression equations and the index is planned to surrender a total score of 100 for the three transformed responses for each formulation. Formulation that measures the highest CI score was selected as the best ranked and optimal one since it is anticipated to fulfill the preset constraints for the three responses [11].

\section{In vitro drug release studies}

Best ranked nanosuspension formulation was subjected further to in vitro drug release evaluation using dialysis bag diffusion technique [12]. $2 \mathrm{ml}$ of the formulation was placed in a dialysis bag (mwt cutoff $300 \mathrm{Da}$, Sigma Aldrich, USA), hermetically sealed and immersed into a $100 \mathrm{ml}$ beaker containing $50 \mathrm{ml}$ of the release media maintained at $37 \pm 0.5^{\circ} \mathrm{C}$ and stirred at $100 \mathrm{rpm}$. Release media was $0.1 \mathrm{~N} \mathrm{HCl}$ for the first $2 \mathrm{~h}, \mathrm{pH} 4.5$ citrate buffer for the next $2 \mathrm{~h}$ and $\mathrm{pH} 7.4$ phosphate buffer for the rest of $24 \mathrm{~h}$ study period. The media incorporated $1 \% \mathrm{v} / \mathrm{v}$ Tween 80 to maintain the sink conditions. Aliquots of $5 \mathrm{ml}$ were withdrawn for drug analysis at predetermined time intervals and immediately replaced with the same volume of fresh media maintained at the same temperature.

\section{Drug assay}

Quantification of the drug in dissolution samples of the release study was performed using a validated International Pharmacopoeia HPLC method of assay [13]. The HPLC comprised of UV detector that set at $216 \mathrm{~nm}$ and LC-10AT vp pump into which an stainless steel column $(25 \mathrm{~cm} \times 4 \mathrm{~mm}$ ) packed with particles of $5 \mu$ octadecylsilyl groups bonded silica gel was attached. A mixture of acetonitrile and water (62:38 respectively) was used as the mobile phase at flow rate of 1.5 $\mathrm{ml} / \mathrm{min}$ and temperature of $30^{\circ} \mathrm{C}$. A series of artemether solutions of known concentrations $(100-10 \mu \mathrm{g} / \mathrm{ml})$ were prepared using the mobile phase as the dilution solution and samples of $20 \mu \mathrm{l}$ were injected into the HPLC (in triplicate). The average area under the drug peak (AP) was determined for each concentration and standard calibration curve for artemether was thus generated and utilized for subsequent determination of the drug in different samples.

\section{Determination of drug release kinetics}

Drug release data of the best ranked drug loaded ethylcellulose nanosuspension was fitted to the power law to determine the drug release kinetics [14]. In this model, fractions of drug release $\leq 0.6$ were evaluated according to the power law seeking best fit value for the diffusion exponent, $n$, that characterizes the drug release kinetics.
Freeze drying and redispersibility of optimal nanosuspension formulation

A $20 \mathrm{ml}$ portion of the optimal nanosuspension formulation was taken in 4 vials and the opening of the vials was covered with tissue paper wrapped by a cotton thread. The vials were then placed inside Dewar flask (Carl Roth Gmbhand Co, Germany) containing liquid nitrogen in order to supercool and freeze. The frozen samples were placed inside $600 \mathrm{ml}$ fast-freeze flask with attached adapter (Labconco®, USA). During the freeze-drying process, temperature was kept about-70 ${ }^{\circ} \mathrm{C}$ and vacuum was fixed at $162 \mathrm{mT}$. After $48 \mathrm{~h}$, lyophilized powder samples were then collected and stored in desiccator for stability studies.

\section{Short term stability studies of optimal nanosuspension formulation}

This study was conducted to explore the effect of reconstitution solution type and storage temperature on stability of the optimal nanosuspension. $10 \mathrm{mg}$ of freeze dried Artemether loaded ethylcellulose nanosuspension powder was reconstituted into $10 \mathrm{ml}$ of either tape water, distilled or double distilled water. Samples were then stored in glass vials for $2 \mathrm{w}$ at $20^{\circ} \mathrm{C}$ (in a control area), 35 ${ }^{\circ} \mathrm{C}$ (in an oven) and $4^{\circ} \mathrm{C}$ (in a freezer). After $2 \mathrm{w}$, samples were visually observed for any sedimentation. The particle size and size distribution was performed using the DLS.

\section{Statistical data analysis}

Average values were considered to present attributes of investigated nanosuspension formulations. Inferential statistics including regression analysis, residual regression, surface model fitting, one sample student $t^{\prime}$ test, analysis of variance (ANOVA) and mathematical approaches were utilized to investigate for the possible single and/or combined influences of different variables examined in this study on the qualifications of the produced nanosuspensions. Mathematical based regression model was used to generate and validate one $\mathrm{CI}$ score ranking of different nanosuspension formulations. Pearson correlation coefficient (r) was used to evaluate the association strength between calibration data and drug concentration for the HPLC method of drug analysis and, moreover, to evaluate the fitting strength of dissolution data to the power law model during determination of diffusion exponent of the drug release. Computations were aided by STATISTICA software package (StatSoft, Inc. version 8, 2007, USA) and in all cases, probability $\mathrm{p} \leq 0.05$ was considered as a cutoff point for significant influences at a confidence limit of 95\%.

\section{RESULTS AND DISCUSSION}

Formulation components and the respective displayed particles' attributes of different artemether loaded ethylcellulose nanosuspension runs within the experimental design are summarized in table 1. Estimated individual and joint influences of artemether: ethylcellulose ratio, content of tween 80 and ratio of chloroform: water volume on different particle attributes of the yielded formulations are abridged in table 2 .

\section{Influences of investigated variables on attributes of nanosuspensions}

From the results included in table 1 and 2, it appears that different variables investigated in this study (drug: polymer ratio, Tween 80 content and chloroform: water volume ratio) have influenced, to a varying extent, the particle size, $\%$ peak intensity (\% IP) and polydisperse index (PDI) characteristics of the produced nanosuspension formulations. These effects will be considered in more details in the following subsections.

\section{Effects on particle size}

Displayed values of particle size within the 18 nanosuspensions are considerably vary in the range $12-465 \mathrm{~nm}(\mathrm{p}=0.0002$, table 1$)$. Particle size and size distribution properties are known to influence saturation solubility, dissolution rate, physical stability and, consequently, the in vivo profile of nanosuspensions [15]. Smaller particle sizes $(10-600 \mathrm{~nm})$ with narrow size distribution (PDI $\leq 0.25)$ are essential parameters that denote the satisfactory homogenity, dispersability, stability and activity of nanosuspensions [16]. Therefore, most, if not all, of produced nanosuspension formulations deem within the nanosized range of particles. 
Table 1: Formulation runs and the relevant particles properties of artemether loaded ethylcellulose nanosuspensions for mixed-level three factors factorial design

\begin{tabular}{|c|c|c|c|c|c|c|}
\hline \multirow[t]{2}{*}{ Runs } & \multicolumn{3}{|c|}{ Variable settings } & \multicolumn{3}{|c|}{ Measured particles properties $^{c}$} \\
\hline & ART: ECa & Tween 80 (g) & C: $\mathbf{W}^{\mathbf{b}}$ & Size (nm) & PI (\%)d & PDIe \\
\hline F1 & $1: 1$ & 0.1 & $1: 5$ & 211 & 84.6 & 1.00 \\
\hline $\mathrm{F} 2$ & $1: 1$ & 0.1 & $1: 10$ & 465 & 46.0 & 1.00 \\
\hline F3 & $1: 2$ & 0.1 & $1: 5$ & 184 & 85.4 & 1.00 \\
\hline $\mathrm{F} 4$ & $1: 2$ & 0.1 & $1: 10$ & 373 & 87.0 & 0.55 \\
\hline F5 & $1: 3$ & 0.1 & $1: 5$ & 200 & 61.9 & 1.00 \\
\hline F6 & $1: 3$ & 0.1 & $1: 10$ & 274 & 91.3 & 0.42 \\
\hline F7 & $1: 1$ & 0.15 & $1: 5$ & 278 & 45.8 & 1.00 \\
\hline F8 & $1: 1$ & 0.15 & $1: 10$ & 206 & 67.0 & 0.93 \\
\hline F9 & $1: 2$ & 0.15 & $1: 5$ & 211 & 89.4 & 0.41 \\
\hline F10 & $1: 2$ & 0.15 & $1: 10$ & 161 & 76.0 & 0.83 \\
\hline F11 & $1: 3$ & 0.15 & $1: 5$ & 221 & 94.5 & 0.36 \\
\hline F12 & $1: 3$ & 0.15 & $1: 10$ & 201 & 92.6 & 0.36 \\
\hline F13 & 1:1 & 0.2 & $1: 5$ & 284 & 49.5 & 0.65 \\
\hline F14 & $1: 1$ & 0.2 & $1: 10$ & 12 & 50.0 & 0.70 \\
\hline F15 & $1: 2$ & 0.2 & $1: 5$ & 302 & 59.0 & 0.97 \\
\hline F16 & $1: 2$ & 0.2 & $1: 10$ & 184 & 59.7 & 0.93 \\
\hline F17 & $1: 3$ & 0.2 & $1: 5$ & 366 & 64.9 & 0.91 \\
\hline F18 & $1: 3$ & 0.2 & 1:10 & 202 & 77.0 & 1.00 \\
\hline
\end{tabular}

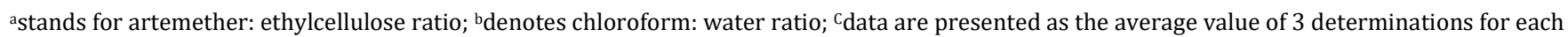
property; dindicates peak intensity and e points to polydisperse index.

Table 2: Effect estimates of investigated variables on the particles attributes of different drug loaded ethylcellulose nanosuspension formulations

\begin{tabular}{lll}
\hline Variables setting & \multicolumn{2}{l}{ Effect estimates on particles properties } \\
\cline { 2 - 3 } & Particle size & \% PI \\
\hline D: P ratio (L) & 1.045 & $-0.235^{*}$ \\
Tween 80 content (L) & $-78.397^{*}$ & -0.162 \\
C: W ratio (L) & 19.889 & -188 \\
Joint D: P ratio and Tween 80 content (L) & -0.017 & -0.012 \\
Joint C: W ratio and Tween 80 content (L) & $-115.103^{* *}$ & -0.022 \\
Joint D: P ratio and C: W ratio & $178.500^{* *}$ & -0.035 \\
\hline
\end{tabular}

D: P ratio and C: W ratio denote artemether: ethylcellulose ratio and chloroform: water ratio, respectively; L represents linear level; PI and PDI stand for peak intensity and polydisperse index, in sequence; ${ }^{*}$ and ${ }^{* *}$ indicate significant effect at $\mathrm{p} \leq 0.05$ and $\mathrm{p} \leq 0.01$, correspondingly.

It might be true that many factors could possibly affect particle size and uniformity of size distribution of nanosuspensions. In that respect and based on magnitude of effect estimates and the associated probability terms for statistical significant influence of investigated variables on particle size shown in table 2, tween 80 measures a considerable influence on particle size either through its individual effect $(p=0.031)$ or through its combined effect with artemether: ethylcellulose or chloroform: water ratios $(\mathrm{p} \leq 0.01$ for both cases). Despite the displayed proportional relation between particle size of nanosuspension and ratios of either artemether: ethyl cellulose or chloroform: water volume, none of the two ratios has revealed an statistically substantial individual influence on particle size although the individual effect of chloroform: water volume ratio on particle size is approximately 19 times that of artemether: ethyl cellulose ratio ( $p>0.05$ for both effects, table 2).

The negative sign associated with the effect estimate of tween 80 content encourages the consideration of an inverse relation between particle size of produced nanosuspensions and content of tween 80 . This, in turn, is supported by fig. 1 in which significant reduction of particle size is evidenced with increasing content of tween 80 from 0.1 to $0.15 \mathrm{~g}(\mathrm{p}=0.022)$. However, there exists a limit beyond which the particle size becomes less sensitive to further increase in content of tween 80 . The inverse proportionality that observed between particle size and content of tween 80 is expected since increasing concentration of tween 80 , as a surfactant, is supposed to maintain efficient reduction in surface tension, facilitating particle coverage, reduce the chance for particles' agglomeration and, consequently, maintain stability of nanoparticles thus produced. It should be stressed, however, that such relation is valid to a limit beyond which no more decrease in particle size is anticipated in response to further increase in surfactant content, which is probably due to occurrence of a saturation point.

Interestingly, the joint influences of tween 80 content with drug: polymer ratio or with chloroform: water volume ratio are more substantial on the accomplish average particle size than the individual influence of tween 80 based on the values of effect estimates and probability significant terms associated with the three variables setting (table 2).

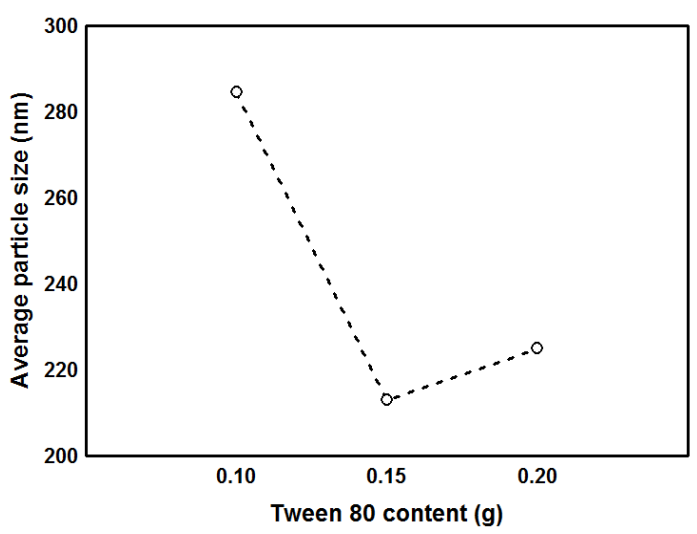

Fig. 1: Changing profile of the average particle size of artemether loaded ethyl cellulose nanosuspension formulations in response to variation in content of tween 80 
The consideration of the joint influences of formulation or processing variables is critical during development of pharmaceutical formulations [17]. In this instance, fig. 2 clearly indicates that reduced particle size is attained at two occasions which are utilization of low content of tween $80(0.1 \mathrm{~g})$ with low drug: polymer ratio (1:3) or using a comparative higher content of tween $80(0.2 \mathrm{~g})$ with a higher drug: polymer ratio (1:1).

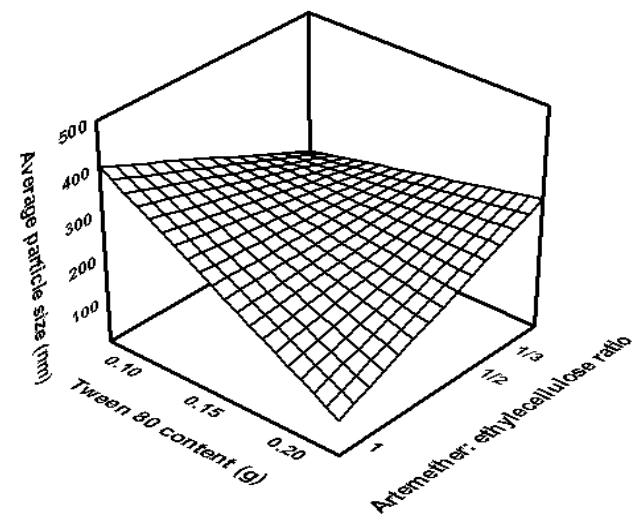

Fig. 2: Surface plot for the changing pattern of average particle size of different nanosuspensions in consequence to joint influence of tween 80 content and drug: polymer ratio

In other words, the interactive setting of both variables determines the desired particle size and, thus, selection of best content of tween 80 to obtain reduced particle size might not be feasible without the simultaneous consideration of the optimum drug: polymer ratio to achieve that particle size. Moreover, the same is applied to the joint influence of tween 80 content and chloroform: water volume ratio.

In another instance, the decrease in particle size observed with increasing artemether: ethylcellulose ratio (decreasing polymer content), though trivial, could possibly be attributed to the reduced viscosity of the internal phase which favors formation of small particles from the drug and polymer droplets [18].

The ratio between external and internal phases of the emulsion can influence the size of dispersed globules and, therefore, the size of produced nanoparticles. The result reveals a proportional influence of the volume ratio of chloroform: water on particle size with a chance to attain average small particles through utilization of a decreased ratio of organic: water volume (1:10). In fact, to obtain small particle, suitable amount of organic solvent is to be available for diffusion in the $\mathrm{o} / \mathrm{w}$ emulsion to prevent coalescence of formed droplets. This suitable amount of organic solvent appears to be achieved with the low rather than high chloroform: water volume ratio, as the result implies.

These findings are in accord with relative published investigational researches on the relation between particle size of nanosuspensions and formulation variables $[10,19]$.

\section{Effects on peak intensity (\% PI)}

Demonstrated peak intensity of different nanosuspension formulations varies between 45.8 and $94.5 \%$ (table 1) and such variation was computed as significant disparity $(\mathrm{p}=0.00003)$. As summarized in table 2 , peak intensity (PI) within different 18 formulations varies inversely with variation in any of the investigated variables at individual or interactive setting. Moreover, only drug: polymer ratio has measured an statistical significant influence on the $\%$ PI property of nanosuspension formulations ( $p=0.037)$. Though the effect estimate of tween 80 content on PI property approximates 13 times that of chloroform: water volume ratio, yet, the influences of both variables are computed as comparable and not statistically sound ( $p=0.096$ and 0.212 for both influences, respectively).

Generally, dynamic light scattering (DLS) techniques provides an intensity weighted distribution, where the contribution of each particle in the distribution relates to the intensity of light scattered by the particle. The relative contribution for very small particles will be proportional to size. Peak intensity represents the mode of particle size distribution and, therefore, it indicates how particles are distributed around the average particle size. The higher the peak intensity \%, the more uniform the distribution of particles around the estimated average size.

The observed increase in \% PI as a result of decreasing drug: polymer ratio (fig. 3) might be attributed to the increased amount of the polymer that aids in formation of sufficient and uniform polymer matrix through which formed nanoparticles are dispersed and trapped, resulting in narrowing of the size distribution of the formed nanoparticles. Nevertheless, narrow distribution with PI of $>90 \%$ is the most desired to denote the uniformity of size distribution around the mean peak size (mode). Thus, only few nanosuspension formulations in the design (F6, F11 and F12) have shown to fulfill the criteria related to PI property (table 1).

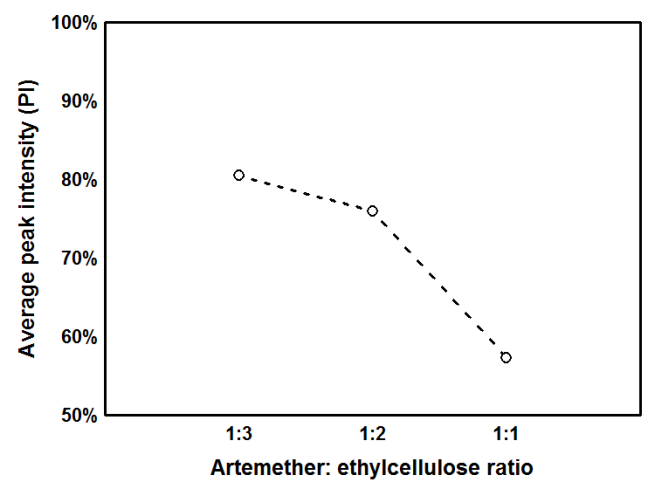

Fig. 3: Alteration pattern of the average peak intensity (\% PI) of artemether loaded ethylcellulose nanosuspension formulations in response to variation in drug: polymer ratio

\section{Effects on polydisperse index (PDI)}

Whilst PI measures size distribution of the formed nanoparticles around the estimated average particle size, polydisperse index (PDI) denotes the physical dispersion pattern of those nanoparticles. In other words, PDI is a reflection to the degree of the system polydispersity where the smaller the PDI value of a nanosuspension $(<0.3)$, the more it would be monodisperse and stable. As appears in table 1, PDI values of different nanosuspension formulations vary significantly between 0.36 and $1(p=0.0002)$ and, moreover, none of the investigated variables has revealed an authenticated influence on PDI property of produced nanosuspensions ( $p>0.05$ for all influences, table 2). Regardless of the statistical ignorance and based on the size of effect estimates of variables on PDI, it might be obvious that the Joint influence of drug: polymer ratio and tween 80 content $(0.296)$ is 1.5 times that of drug: polymer ratio, 4 times that of chloroform: water ratio and 17 times that of tween 80 content (table 2).

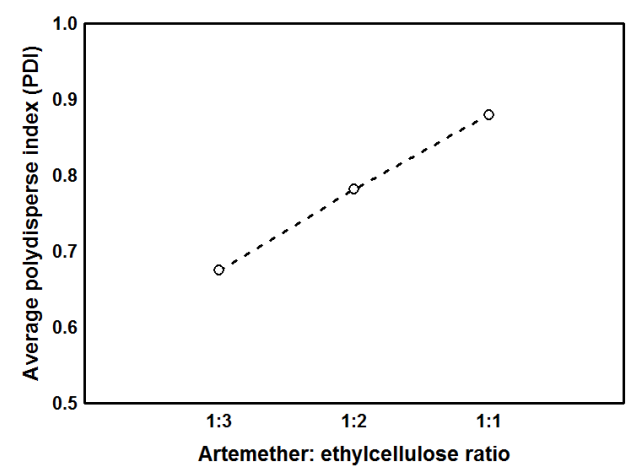

Fig. 4: Variation profile of particles' PDI in consequence to changes in drug: polymer ratio 
The desired decline of PDI recognized with the decreasing of drug: polymer ratio (fig. 4) may be attributed to the fact that as the polymer concentration increased, the amount of nanoparticles per unit volume will be small which would favor the formation of desired monodisperse system (small PDI) as a result of disallowing particle agglomeration.

\section{Formulation optimization by composite index ranking}

Attained transformed values, score of composite index (CI) and ranking order for different artemether loaded ethylcellulose nanosuspension formulations are enclosed in table 3 . The composite index score for each formulation is the sum of 3 transformed properties (particle size, \% PI and PDI) which are constrained to fulfill specific criteria as previously described in the methods section of this manuscript.

Based on the utilized production technique and the intended application, an ideal nanosuspension is anticipated to show smaller nanosized particles, \% PI that approaches $100 \%$ and PDI $\leq 0.25$ [16] However, like other delivery systems, a nanosuspension with particle size that suites all therapeutic applications doesn't exist as has been previously declared [1]. Thus, selection of the constraints for the three properties of the nanosuspensions was aimed at selecting nanosuspension with the most satisfactory particle size, size distribution and dispersion properties. This is because these properties are indicators for dosage form stability and drug delivery efficiency.

Table 3: Composite index and ranking order of artemether loaded ethyl cellulose nanosuspensions based on preset constraints for particle size, peak intensity (\% PI) and polydisperse index (PDI) associated with different formulations

\begin{tabular}{|c|c|c|c|c|c|}
\hline \multirow[t]{2}{*}{ Runs } & \multicolumn{3}{|c|}{ Transformed responses } & \multirow[t]{2}{*}{ CI* } & \multirow[t]{2}{*}{ Rank order } \\
\hline & Particle size & $\%$ PI & PDI & & \\
\hline F1 & 32.31 & 00.00 & 00.00 & 32.31 & 8 \\
\hline $\mathrm{F} 2$ & 0.00 & 00.00 & 00.00 & 00.00 & 18 \\
\hline F3 & 29.74 & 00.89 & 00.00 & 30.63 & 9 \\
\hline F4 & 04.62 & 04.44 & 00.00 & 09.06 & 15 \\
\hline F5 & 32.47 & 00.00 & 00.00 & 32.47 & 7 \\
\hline F6 & 21.54 & 14.00 & 05.00 & 40.54 & 4 \\
\hline F7 & 20.86 & 00.00 & 00.00 & 20.86 & 12 \\
\hline F8 & 33.16 & 00.00 & 00.00 & 33.16 & 5 \\
\hline F9 & 32.31 & 09.78 & 06.67 & 48.76 & 3 \\
\hline F10 & 25.81 & 00.00 & 00.00 & 25.81 & 11 \\
\hline F11 & 30.60 & 21.11 & 15.00 & 66.71 & 1 \\
\hline F12 & 32.64 & 16.89 & 15.00 & 64.53 & 2 \\
\hline F13 & 19.83 & 00.00 & 00.00 & 19.83 & 13 \\
\hline F14 & 00.34 & 00.00 & 00.00 & 00.34 & 17 \\
\hline F15 & 16.76 & 00.00 & 00.00 & 16.76 & 14 \\
\hline F16 & 29.74 & 00.00 & 00.00 & 29.74 & 10 \\
\hline F17 & 05.82 & 00.00 & 00.00 & 05.82 & 16 \\
\hline F18 & 32.81 & 00.00 & 00.00 & 32.81 & 6 \\
\hline
\end{tabular}

${ }^{*}$ Denotes composite index score

Different formulations measure CI score that ranged 0-66.7 and among different formulations investigated, obviously two formulations (F11 and F12) have achieved most advanced CI scores and ranking orders (table 3). Both formulations fabricated with decreased drug: polymer ratio (1: 3$)$ and medium content of tween 80 (0.15 g), however, they differ in the utilized ratios of chloroform: water volume (table 1). This, in turn, signifies the determining role of drug: polymer ratio on \% PI and PDI properties and, moreover, reflects the influential impact of content of tween 80 on the achieved particle size as discussed before. Accordingly, formulation F11 was selected as the optimal formulation that best fulfills the preset constraints for nanosuspension. The findings imitate the effectiveness of $\mathrm{CI}$ method in assortment and ranking of different formulations based on the preset constraints.

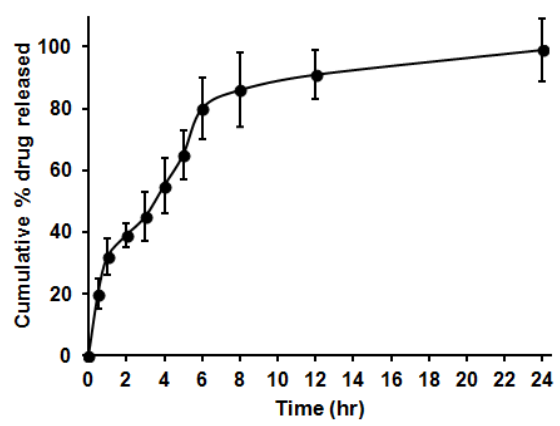

Fig. 5: Drug release profile of artemether from the optimal drug loaded ethyl cellulose nanosuspension formulation. Each data point is the average value of 3 determinations with error bars indicating \pm standard deviation (SD)

\section{In vitro drug release and release kinetics characterization}

Artemether release of the optimal nanosuspension formulation (F11) is depicted in fig. 5. The formulation seems capable to sustain the release of the loaded drug over $24 \mathrm{~h}$.

The burst drug release observed during the initial $2 \mathrm{~h}$ of the release study (fig. 5) could be attributed to the presence of nanosized drug on the surface of the nanoparticles with enhanced dissolution velocity as a consequence of the increased surface area and saturation solubility [20]. On the other hand, the sustained drug release profile over the study duration could be interpreted in terms of the retarding influence of ethylcellulose as water insoluble polymer. It is worth mentioning that ethylcellulose loaded artemether nanosupension offer not only enhancement of drug solubility but also a chance for extending the drug release over prolonged period of time which is a necessary criteria for a successful controlled release drug delivery system.

The average value of the diffusion exponent (n) that estimated from power law for the optimal drug loaded ethylcellulose nanosuspension formulation is computed as $0.438 \pm 0.103$, indicating that the drug release mechanism follows the pure Fickian diffusion where the drug release depends on diffusion through the formed gel of ethylcellulose rather than the drug solubility.

\section{Stability of the optimal drug loaded ethyl cellulose nanosuspension}

Values of particle size and PDI displayed by optimal nanosuspension formulation after reconstitution in different types of water and store for $2 \mathrm{w}$ at different temperature are summarized in table 4. According to the findings of stability study, there are trivial changes in stability of nanoparticles in terms of particle size and polydisperse index in consequence to variation in storage temperature $(\mathrm{p}=0.857)$. 
Table 4: Stability data for the optimal artemether loaded ethylcellulose nanosuspension formulation

\begin{tabular}{|c|c|c|c|c|}
\hline \multirow[t]{2}{*}{ Time period } & \multicolumn{2}{|l|}{ Storage conditions } & \multicolumn{2}{|c|}{ Particles properties $^{a}$} \\
\hline & Reconstitution solvent & Temperature & Particle size (nm) & PDI $^{b}$ \\
\hline \multicolumn{5}{|l|}{ Initially } \\
\hline & Tap water & $30^{\circ} \mathrm{C}$ & 245 & 0.38 \\
\hline & Distilled water & $30{ }^{\circ} \mathrm{C}$ & 232 & 0.31 \\
\hline & Double distilled water & $30{ }^{\circ} \mathrm{C}$ & 230 & 0.30 \\
\hline \multicolumn{5}{|c|}{ After two weeks } \\
\hline & Tap water & $5^{\circ} \mathrm{C}$ & 250 & 0.40 \\
\hline & Tap water & $20^{\circ} \mathrm{C}$ & 246 & 0.41 \\
\hline & Tap water & $35^{\circ} \mathrm{C}$ & 247 & 0.35 \\
\hline & Distilled water & $5^{\circ} \mathrm{C}$ & 240 & 0.41 \\
\hline & Distilled water & $20^{\circ} \mathrm{C}$ & 243 & 0.34 \\
\hline & Distilled water & $35^{\circ} \mathrm{C}$ & 237 & 0.36 \\
\hline & Double distilled water & $5^{\circ} \mathrm{C}$ & 244 & 0.34 \\
\hline & Double distilled water & $20^{\circ} \mathrm{C}$ & 235 & 0.36 \\
\hline & Double distilled water & $35^{\circ} \mathrm{C}$ & 236 & 0.33 \\
\hline
\end{tabular}

adata are presented as the average value of 3 determinations for each property; bindicates polydisperse index.

The effect of the type of reconstitution solvent on particle size during storage is more profound than that measured on particles PDI. In fact, the increase in aggregation tendency when dissolving nanoparticles in tap water (table 4) might be explained by the presence of ions in tap water which might cause a reduction in thickness of the electric double layer, allowing for greater particle-particle interaction and thereby increasing level of aggregation [21]. On the other hand, the observed increase in particle size in distilled water and in double distilled water can be explained by the increase in Ostwald ripening in which larger particles grow at the expense of the smaller particles due to the wellknown Kelvin effect [22].

The visual observation has indicated that no crystal growth was noted during the period of study and no changes in appearance or settling behavior of the freeze dried ethylcellulose nanosuspension when observed visually. Consequently, these findings support the assumption that the nanosuspension formulation is physically stable, at least under the setting conditions of the present stability study.

\section{CONCLUSION}

Mixed levels and composite index designs were both proved efficient in screening and optimization of artemether loaded ethylcellulose nanosuspension formulations. Drug: polymer ratio, content of surfactant and organic: water volume ratio were found to affect particle size of produced nanosuspensions, either through their individual or joint influences. Peak intensity property of nanosuspensions was found to be influenced by drug: polymer ratio whereas the influences of different variables on the polydisperse index property appear inconsequential. Optimal Artemether nanosuspension proved stable and capable to improve and maintain the release of loaded drug over $24 \mathrm{~h}$, at least under the setting conditions of the study.

\section{ACKNOWLEDGMENT}

Authors would like to admit Prof. Peter Wich (Department of Pharmaceutical Chemistry, University of Mainz, Germany) and Prof. Peter R. Langguth (Department of pharmaceutical technology and Biopharmaceutics, University of Mainz, Germany) for making accessible of most of the instruments and devices utilized in this study.

\section{AUTHORS CONTRIBUTION}

All authors share equal contribution to the presented manuscript.

\section{CONFLICT OF INTERESTS}

\section{Declared none}

\section{REFERENCES}

1. Muller RH, Jacobs C, Kayser O. Nanosuspensions as particulate drug formulations in therapy: rationale for development and what we can expect for the future. Adv Drug Delivery Rev 2001;47:3-19.
2. Patel VR, Agrawal YK. Nanosuspension: an approach to enhance solubility of drugs. J Adv Pharm Tech Res 2011;2 Suppl 2:81-7.

3. Silamut K, Newton PN, Teja-Isavadharm P, Suputtamongkol Y, Siriyanonda D, Rasameesoraj $\mathrm{M}$, et al. Artemether bioavailability after oral or intramuscular administration in uncomplicated falciparum malaria. Antimicrob Agents Chemother 2003;47 Suppl 12:3795-8.

4. Laxmi M, Bhardwaj A, Mehta S, Mehta A. Development and characterization of nanoemulsion as carrier for the enhancement of bioavailability of artemether. Artif Cells Nanomed Biotechnol 2015;43 Suppl 5:334-44.

5. Anna B, Harisha KM, Uday K. Development, charecterization and evaluation of solid dispersion of artemether and Lumefantrine by solvent evaporation method using hydrophilic polymers. Int J Pharm Pharm Sci 2010;6 Suppl 2:180-5.

6. Somasundaram I, Sathesh Kumar S. Pramipexole dihydrochloride loaded MPEGPCL nanosuspension by modified nanoprecipitation: in vitro and in vivo evaluation. Asian J Pharm Clin Res 2016;9 Suppl 6:1-7.

7. Kilor V, Sapkal N, Daud A, Humne S, Gupta T. Development of stable nanosuspension loaded oral films of glimepiride with improved bioavailability. Int J Appl Pharm 2017;9 Suppl 2:28-33.

8. George M, Ghosh I. Identifying the correlation between drug/stabilizer properties and critical quality attributes (CQAs) of nanosuspension formulation prepared by wet media milling technology. Eur J Pharm Sci 2013;48 Suppl 1-2:142-52.

9. Bonhoeffer $B$, Kwade $A$, Juhnke $M$. Impact of formulation properties and process parameters on the dispensing and depositioning of drug nanosuspensions using micro-valve technology. J Pharm Sci 2017;106 Suppl 4:1102-10.

15. Sarmaha JK, Mahantab R, Bhattacharjeea SK, Ranadeep M, Angshuman B. Controlled release of tamoxifen citrate encapsulated in cross-linked guar gum nanoparticles. Int J Biol Macromol 2011;49:390-6.

16. Derringer G, Suich R. Simultaneous optimization of several response variables. J Qual Technol 1980;12 Suppl 4:214-9.

17. Kumar R, Nagarwal RC, Dhanawat M, Pandit JK. In vitro and in vivo study of indomethacin loaded gelatin nanoparticles. J Biomed Nanotechnol 2011;7 Suppl 3:325-33.

18. International Pharmacopoeia, Artemether assay specific monograph. 6th edition, Geneva: WHO publications; 2016. Available from: http://apps.who.int/phint/2016/ index.html\#d/b.6.2.2.16. [Last accessed on 10 Feb 2017]

19. Korsmeyer RW, Gurny R, Doelker E, Buri P, Peppas NA. Mechanisms of solute release from porous hydrophillic polymers. Int J Pharm 1983;15:25-35.

20. Liversidge GG, Cundy KC. Particle size reduction for improvement of oral bioavailability of hydrophobic drugs: absolute oral bioavailability of nanocrystalline danazol in beagle dogs. Int J Pharm 1995;125:91-7.

21. Chen Y, Liu J, Yang X, Zhao X, Xu H. Oleanolic acid nanosuspensions: preparation, in vitro characterization and enhanced hepatoprotective effect. J Pharm Pharmacol 2005;57:259-64. 
22. Nur AO, Elamin KM, Osman ZA. Individul and mutual effects of applied compression force, mixing time and manufacturing method on properties of HPMC oral matrices. World J Pharm Pharm Sci 2015;4 Suppl 6:1327-44.

23. Dinarvand R, Mirfattahi S, Atyabi F. Preparation, characterization and in vitro drug release of isosorbide dinitrate microspheres. J Microencapsul 2002;19 Suppl 1:73-81.

24. Kalimuthu S, Yadav AV. Formulation and evaluation of carvedilol loaded Eudragite 100 nanoparticles. Int J PharmTech Res 2009;1 Suppl 2:179-83.

25. Rao KR, Senapati P, Das MK. Formulation and in vitro evaluation of ethyl cellulose microspheres containing zidovudine. J Microencapsul 2005;22 Suppl 8:863-76.
26. Zimmermann E, Müller RH, Mäder K. Influence of different parameters on reconstitution of lyophilized SLN. Int J Pharm 2000;196 Suppl 2:211-3.

27. Verma S, Kumar S, Gokhale R, Burgess DJ. Physical stability of nanosuspensions: Investigation of the role of stabilizers on Ostwald ripening. Int J Pharm 2011;406 Suppl 1-2:14552 .

\section{How to cite this article}

- $\quad$ Nasrin E Khalifa, Abubakr O Nur, Zuheir A Osman. Artemether loaded ethylcellulose nanosuspensions: effects of formulation variables, physical stability and drug release profile. Int J Pharm Pharm Sci 2017;9(6):90-96. 